\title{
TIME-AVERAGED TURBULENT MIXING AND VERTICAL CONCENTRATION DISTRIBUTION OF HIGH-DENSITY SUSPENSIONS FORMED UNDER WAVES
}

\author{
Bing Yan ${ }^{1}$, Qing-He Zhang ${ }^{2}$, Michael P. Lamb ${ }^{3}$
}

\begin{abstract}
We analyzed oscillating flow data from U-tube experiments by Lamb et al. (2004) and found that the time-averaged turbulent kinetic energy (TKE) near bed decreased exponentially with height above the bed in high-densitysuspension (HDS) flows under waves, and that the ratios of TKE distributions in the streamwise, cross-stream, and vertical dimensions were constant. Based on the finite-mixing-length theory, a semi-theoretical time-averaged suspended sediment concentration model for HDS was developed. To avoid the stability problems with the numerical solution, a simplified model was also formulated through combing the apparent Fickian diffusivity and the damping function. The comparison between the calculated results and measurements shows both models consider the effect of the sediment-induced stratification well.
\end{abstract}

Keywords: high density suspension; vertical concentration distribution; turbulent mixing; turbulent kinetic energy; waves; silt

\section{INTRODUCTION}

During storm events, navigation channels on silty coast often suffer from rapid siltation. This rapid siltation is attributed to the occurrence of high-density suspensions (HDS) near bottom due to waves (Zhao 2006). Hou (2004) found that the concentration of HDS near bed was higher than $10 \mathrm{~g} / \mathrm{l}$ and up to $60 \sim 80 \mathrm{~g} / 1$ on a beach around Huanghua Harbor under winds of $17 \sim 21 \mathrm{~m} / \mathrm{s}$. The measured thickness of HDS in the field was about $0.5 \sim 1.0 \mathrm{~m}$. The laboratory experiments were also preformed in a wave tank (Han 2005) to investigate the phenomena of HDS, which showed that there existed an obvious vertical concentration gradient even though the concentration near bed was lower than $10 \mathrm{~g} / \mathrm{l}$ in the small-scale tank. This seems to be consistent with the conclusion that appreciable stratification is generated by sediment-induced buoyancy effects at fairly low suspended sediment concentrations (a few $0.1 \mathrm{~g} / \mathrm{l}$ ) in turbulent open-channel flow (Winterwerp 2006).

Recently experiments on turbulent wave boundary layers over silt-dominated beds have been performed using a U-tube (Lamb et al. 2004; Lamb and Parsons 2005). The experimental wave orbital velocities $(15 \sim 60 \mathrm{~cm} / \mathrm{s})$ and periods $(3 \sim 8 \mathrm{~s})$, as well as the characteristics of the HDS (near-bed sediment concentration ranging from $17 \sim 80 \mathrm{~g} / 1$ ), were comparable to field observations during storm.

For further explanation and prediction of rapid channel siltation on silty coast, based on Lamb's experiments, a semi-theoretical model to characterize the time-averaged turbulent mixing and vertical concentration distribution of HDS formed under waves will be presented through the finite-mixinglength theory (Nielsen and Teakle 2004) in the present paper.

\section{THE SEMI-THEORETICAL MODEL}

\section{The Finite-mixing-length Theory}

According to Nielsen and Teakle (2004), the time-averaged mass concentrations of suspended sediment $c(z)$ result from the balance between an upward mixing flux $q_{m}$ and a downward settling flux.

$$
q_{m}-w_{s} c(z)=0
$$

where $w_{s}$ is the settling velocity, $z$ is the vertical coordinate (the zero point is on the bed). The swapping of fluid parcels (including suspended sediment) between different levels can generate a net vertical flux of sediment. The sediment concentrations in the lower and upper parcels are $c(z-l / 2)$ and $c(z-l / 2)$ respectively, where $l$ is the mixing length. If the parcels travel vertically with equal and opposite velocities $\pm w_{m}$, the resulting sediment flux density is (Nielsen and Teakle 2004)

$$
q_{m}=w_{m}[c(z-l / 2)-c(z+l / 2)]
$$

By Taylor expansion

\footnotetext{
${ }^{1}$ Tianjin Research Institute of Water Transport Engineering \& Key Laboratory of Engineering Sediment of Ministry of Transport, Tianjin, 300456, PR China, Email: ybing2000@163.com

${ }^{2}$ School of Civil Engineering, Tianjin University \& Key Laboratory of Harbor and Ocean Engineering (Tianjin University), Ministry of Education, Tianjin, 300072, PR China, Corresponding-Email: qhzhang@tju.edu.cn

${ }^{3}$ Division of Geological and Planetary Sciences, California Institute of Technology, MC 170-25, 1200 E. California Blvd., Pasadena, CA 91125, USA, Email: mpl@gps.caltech.edu
} 


$$
q_{m}=-w_{m} l\left(\frac{d c}{d z}+\frac{l^{2}}{24} \frac{d^{3} c}{d z^{3}}+\mathrm{L}\right)
$$

With Eq. 3, Eq. 1 becomes

$$
-w_{m} l\left(\frac{d c}{d z}+\frac{l^{2}}{24} \frac{d^{3} c}{d z^{3}}+\mathrm{L}\right)-w_{s} c=0
$$

By including only the first two terms of the Taylor expansion, we obtain

$$
\frac{l^{2}}{24} \frac{d^{3} c}{d z^{3}}+\frac{d c}{d z}+\frac{w_{s}}{w_{m} l} c=0
$$

Eq. 5 needs to be solved numerically as a boundary value problem. The mixing velocity $w_{m}$ and the mixing length $l$ are key parameters to determinate the sediment concentration profile in Eq. 5. Assuming that the mixing velocity distribution is proportional to the turbulent velocity fluctuations, Nielsen and Teakle (2004) gave

$$
w_{m}(z)=w_{m}(0) \exp \left(-z / L_{w}\right)
$$

where $L_{w}$ is a vertical scale. Absi (2006) proposed that $L_{w}$ equals to the wave boundary layer thickness and $w_{m}(0)=\gamma u_{*}$, where $u_{*}$ is the friction velocity and $\gamma$ is a coefficient $(=0.4)$ which has been analyzed by Nielsen and Teakle (2004). Based on law-of-the-wall reasoning Nielsen and Teakle (2004) adopted a mixing length distribution which is proportional to height above the apparent bed level.

$$
l=\lambda^{\prime} z
$$

where $\lambda^{\prime}$ is a coefficient which was chosen as $\lambda^{\prime}=1$.

However, the above mixing velocity and mixing length formulae were derived in the condition of low suspended sediment concentration. In order to describe the damping of turbulence and sedimentinduced stratification in HDS, we will focus on modification of the above expressions by incorporating the effects of HDS.

\section{Thickness of High-Density Suspensions}

Firstly the thickness of HDS needs to be defined. HDS typically have sediment concentration in excess of $10 \mathrm{~g} / \mathrm{L}$ and are defined by the presence of a lutocline (Lamb et al. 2004). The height of HDS has not been modeled extensively. The existing models generally used some hydrodynamic characteristics or reference concentration to characterize the lutocline. Lamb and Parsons (2005) analyzed several existing models and found that they have not shown the predictive capability for all of their laboratory experiments. In fact, the lutocline is an abrupt change in vertical concentration gradient. If it is hypothesized that the time-averaged vertical concentration distribution $c$ is a smooth function, we consider the position of the maximum curvature in $c$ as the upper boundary of HDS, the thickness of HDS $\delta_{H}$ can be given as (see Fig. 1)

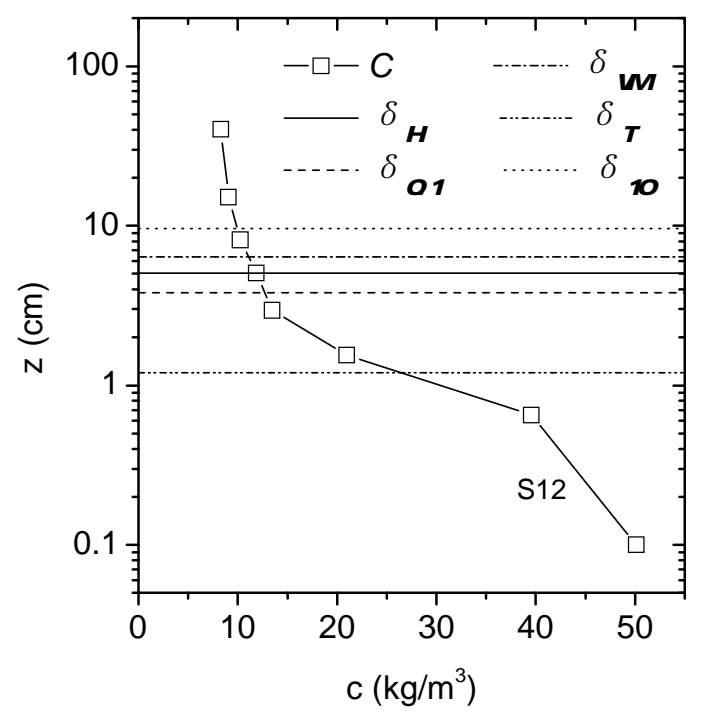

Figure 1. Comparison between the lutocline height $\delta_{H}$ with measured concentration in experiment $\mathrm{S} 12$ of Lamb et al. (2004) and some models' heights calculated by $\delta_{V M}, \delta_{T}, \delta_{0.1}$ and $\delta_{10}$. 


$$
\delta_{H}=\left\{z \mid \max \left[\frac{c^{\prime \prime}(z)}{\left(1+c^{\prime}(z)^{2}\right)^{3 / 2}}\right], 0 \leq z \leq h\right\}
$$

where $h$ is the water depth.

The lutocline height $\delta_{H}$ was compared with measured concentration in experiment S12 from Lamb et al. (2004) as well as some models' heights calculated from $\delta_{V M}$ (Vinzon and Mehta 1998), $\delta_{T}$ (Traykovski et al. 2000), $\delta_{0.1}$ (elevation where the concentration $=0.1 c_{\text {bed }}, c_{\text {bed }}$ is the near-bed sediment concentration), $\delta_{10}$ (elevation where the concentration $=10 \mathrm{~g} / \mathrm{l}$ ). The definition in this study $\delta_{H}$ seems to have a more reasonable result from visual observation in Fig. 1. Because $c$ is unknown, $\delta_{H}$ need to be obtained iteratively during concentration solution.

\section{The Mixing Velocity}

Turbulence dominates the motion of sediments and keeps them in suspension in the near-bed layer under waves. Thereby it is instructive to relate mixing velocity of suspended sediment to the vertical turbulent fluctuations. Nezu and Nakagawa (1993) assumed that the total turbulent intensity $K$ decreases exponentially with $z$ in the fresh open-channel flow. The measured values of $\sqrt{K}$ at each experiment with HDS of Lamb et al. (2004) were fitted to the exponential distribution

$$
\sqrt{K}=u_{0} \exp \left(-z / L_{t}\right)
$$

where $u_{0}$ is the characteristic turbulent velocity extrapolated to the bottom at $z=0$ and $L_{t}$ is the vertical length scale.

Through analyzing experimental data, the time-averaged turbulent intensity with HDS near bed under waves can also be characterized as decreasing exponentially with $z$. The characteristic turbulent velocity that accounts for stratification effects $u_{0}$ can be expressed as a function of the friction velocity $u_{*}$ in fresh water and the mean mass concentration of HDS $C_{m}$ (see Fig. 2), namely

$$
u_{0}=u_{*_{w}} \exp \left(-\alpha_{1} \frac{\rho_{s}-\rho_{0}}{\rho_{s} \rho_{0}} C_{m}\right)
$$

where $\alpha_{I}$ is a constant $(=70), \rho_{s}$ and $\rho_{0}$ are the density of sediment and clear water respectively. The friction velocity $u_{0}$ affected by sediment-induced stratification is usually less than the friction velocity $u_{*_{w}}$ in fresh water. When there is no suspended sediment in water, $C_{m}=0$ and then $u_{0}=u_{*_{w}}$.

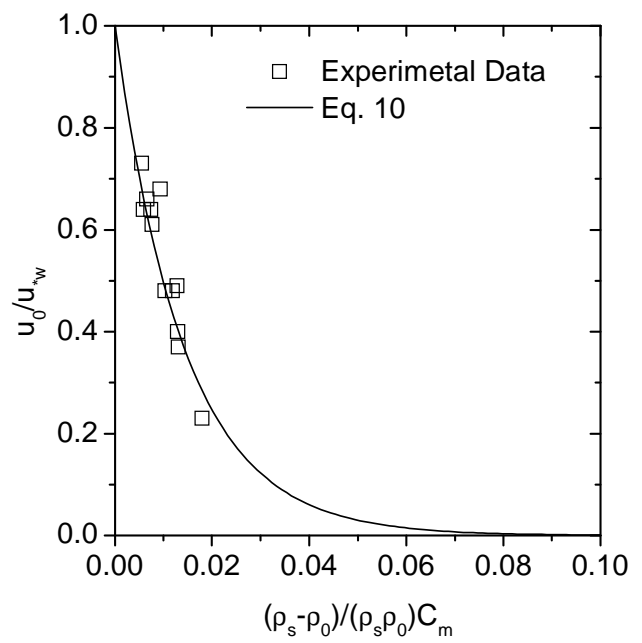

Figure 2. $u_{0}$ as a function of $u_{\mathrm{w}}$ and $C_{m}$ ( $C_{m}$ were calculated by Eq. 8 using the experiment S4 S15 of Lamb et al. (2004)).

The wave friction velocity $u_{*_{w}}$ in fresh water is determined by

$$
u_{* w}=\left(0.5 f_{w} u_{w}^{2}\right)^{0.5}
$$

where $u_{w}$ is the wave orbital velocity amplitude at sea bed, $f_{w}$ is the wave friction factor, which is calculated using the model of Swart (1974), as

$$
f_{w}=\exp \left[5.213\left(2.5 k_{s} / A\right)^{0.194}-5.977\right]
$$


where $A$ is the orbital amplitude of wave motion at the bed, $k_{s}$ is the bed roughness, which is given by

$$
k_{s}=20 \eta^{2} / \lambda+5 \theta^{\prime} d_{50}
$$

where $\eta$ is the sand ripple height, $\lambda$ is the sand ripple length, $d_{50}$ is the median diameter, $\theta^{\prime}$ is the skin friction Shields parameter (Nielsen 1992).

The vertical length scale $L_{t}$ equals to the thickness of boundary layer $\delta_{w}$ approximately, which is calculated by (You 1991).

$$
L_{t} \approx \delta_{w}=\frac{2 \kappa u_{* w}}{\omega}
$$

where $\kappa=0.4$ is von Karman's constant, $\omega$ is the wave angular frequency.

If $u^{\prime}, v^{\prime}$ and $w^{\prime}$ are the velocity fluctuations in the $x$ (streamwise) direction, $y$ (spanwise) direction and $z$ (vertical) direction respectively, the turbulent kinetic energy (TKE) is calculated by

$$
K=\frac{1}{2}\left(\overline{u^{\prime 2}}+\overline{v^{\prime 2}}+\overline{w^{\prime 2}}\right)
$$

Nezu and Nakagawa (1993) argued that the ratios of distribution of the turbulent energy are constant in the fresh open-channel flow.

$$
\overline{u^{\prime 2}} /(2 K)=0.55, \overline{v^{\prime 2}} /(2 K)=0.28, \overline{w^{\prime 2}} /(2 K)=0.17
$$

This indicates that universality and similarity exit in the structure of the turbulence and the ratios of distribution in three directions do not vary in the different water depths. The following question is whether the ratios are still constant in the near-bed water under waves with HDS. We reanalyzed the measured data of experiments and found the answer was positive. Fig. 3 shows the relations between the velocity fluctuations variances which are approximated as

$$
\overline{u^{\prime 2}} /(2 K)=0.53, \overline{v^{\prime 2}} /(2 K)=0.38, \overline{w^{\prime 2}} /(2 K)=0.09
$$
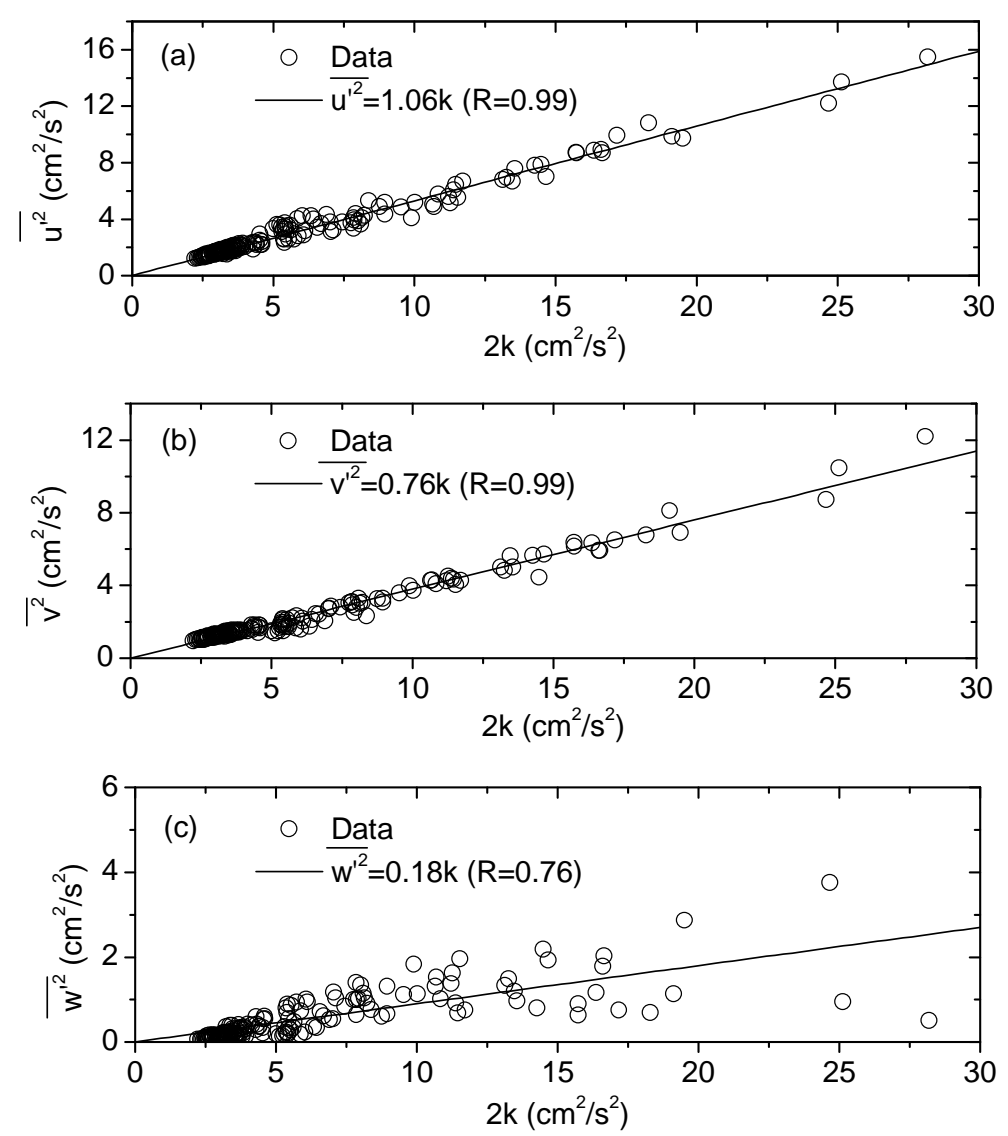

Figure 3. Relations of the velocity fluctuations $u^{\prime}, v^{\prime}$ and $w^{\prime}$ to TKE in sediment suspended flows (experiment S1 S15 of Lamb et al. (2004)).

Thus the vertical mixing velocity $w_{m}$ can be presented as 


$$
w_{m}=\sqrt{\overline{w^{\prime 2}}}=\gamma^{\prime} \sqrt{K}=\gamma^{\prime} u_{*_{w}} \exp \left(-\alpha_{1} \frac{\rho_{s}-\rho_{0}}{\rho_{s} \rho_{0}} C_{m}\right) \exp \left(-\frac{z}{\delta_{w}}\right)
$$

where $\gamma^{\prime}$ is determined by experiments $(\approx 0.4)$. This coincides with the hypothesis in the water with low suspended sediment (Eq. 6). In clear water flows, $C_{m}=0$ and then Eq. 18 reduces to Eq. 6. Fig. 4 shows the comparison between measurement of vertical mixing velocity in Experiment S4 (Lamb et al. 2004) and the calculated values by Eq. 18. The good agreement indicates that the equation reflects the vertical distribution of mixing velocity with HDS. Thus, the universality and similarity of the turbulent structure also exits in the near-bed water with HDS formed under waves.

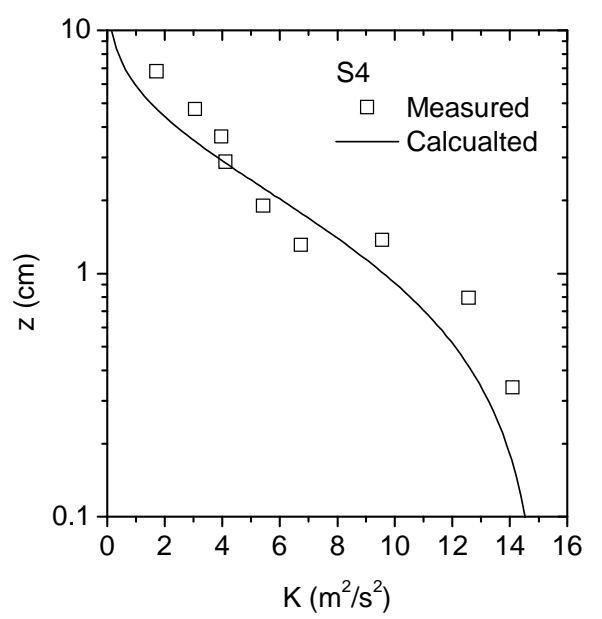

Figure 4. Comparison between measured and calculated results of vertical mixing velocity. Data is from experiments S4 of Lamb et al. (2004).

\section{The Mixing Length}

When the stratification is strong, the mixing length is limited because there is a substantial suppression of vertical motion. The mixing length $l^{\prime}$ in a stratified and turbulent fluid was derived by Balmforth et al. (1998) as

$$
l^{\prime}(z)=\frac{l K^{1 / 2}}{\left(K+r l^{2} b_{z}\right)^{1 / 2}}
$$

where $b_{z}$ is the buoyancy frequency and $r$ is a non-dimensional parameter. So the mixing length has two limits: when there is not stratification, $l^{\prime}$ reaches the maximum $l^{\prime}=l$; when there is a strongly stratified limit, $l^{\prime}$ reaches the minimum $l^{\prime} \approx\left(K / r b_{z}\right)^{1 / 2}$.

\section{Solution of Model}

We have determined the mixing velocity and length using Eq. 18 and Eq.19. Combined with Eq. 5 and an equation for settling velocity (Lewis et al. 1949)

$$
\omega_{s}=\omega_{s 0}\left(1-c_{V}\right)^{n}
$$

where $\omega_{s 0}$ is terminal settling velocity of a solitary particle in a fluid, $c_{V}$ is sediment concentration by volume, $n$ is an exponent, which is determined by Cheng (1997). The resulting semi-theoretical model is a third-order differential equation problem and needs to be solved numerically as a boundary value problem. Three boundary conditions are (1) the reference concentration near bed (using the nearest-bed measured values), (2) the concentration on the top of the tube is zero, and (3) the gradient of concentration on the top of the tube is zero.

\section{A SIMPLIFIED MODEL}

The stability of the numerical solution for Eq. 5 sometimes suffers from oscillations. For easy application of the solution, a simplified model is necessary.

The finite-mixing-length model characterizes the mixing process through the mixing velocity and mixing length, but not through diffusivity like traditional models based on the Fickian diffusion theory. From discussions above, it is known that the sediment-induced stratification in HDS makes the strength of turbulence weak (which leads to a slower mixing velocity) and a shorter mixing length. Both cause the diffusivity within HDS to be less than that in clear water flows. Here we combined the finite- 
mixing-length theory with the traditional model. The damping function of van Rijn's model (2007) was adopted to reflect the effect of stratification through modifying the apparent Fickian diffusivity derived from the finite-mixing-length theory. In this way, we not only take advantage of the finite-mixing-length theory, but also simplify the calculation.

According to the 'locally-homogenous approximation', the apparent Fickian diffusivity $\varepsilon_{F i c k}$ was derived by Teakle (2006) as

where $w_{m}$ is calculated by Eq. 6 .

$$
\varepsilon_{F i c k}=\frac{\omega_{s} l}{2 \sinh ^{-1}\left(\frac{\omega_{s}}{2 w_{m}}\right)}
$$

The damping function $\Phi_{d}$ from van Rijn (2007) is

$$
\phi_{d}=\phi_{f s}\left[1+\left(c_{V} / c_{g e l, s}\right)^{0.8}-2\left(c_{V} / c_{g e l, s}\right)^{0.4}\right]
$$

where $\Phi_{f s}=d_{50} / 1.5 d_{\text {sand }}$ is an additional calibration factor and $\Phi_{f s}=1$ for $d_{50} \geq 1.5 d_{\text {sand }}, d_{\text {sand }}=62 \mu \mathrm{m}$, and $c_{g e l, s}=6.5$ is maximum bed concentration by volume.

With Eq. 21 and Eq.22, the governing equation is written as

$$
\phi_{d} \varepsilon_{\text {Fick }} \frac{\partial c}{\partial z}+\omega_{s} c=0
$$

This is a first-order differential equation, which can be solved by numerical iteration.

\section{RESULTS AND DISCUSSION}

Numerical solutions from the semi-theoretical model (Eq. 5 and Eq. 18 20) are compared with measurements from experiments by Lamb and Parsons (2005). From Fig. 5, the results show that the model reproduces the results of laboratory experiments and reflects the mechanism of turbulent mixing of high-density suspensions. The model underestimates the suspended sediment concentration above $0.2 \mathrm{~m}$. The reason may be that the width of the U-tube in the spanwise direction is just $0.2 \mathrm{~m}$ and the water above $0.2 \mathrm{~m}$ is influenced by the lateral walls, from which the additional turbulence increases the suspended sediment concentration. However we did not consider the factor of the lateral walls in the semi-theoretical model. The experiments also had contamination of TKE from mixing in the endtanks, which resulted in higher than expected suspended sediment concentrations in the upper water column (Lamb and Parsons 2005).

The median diameters of suspended sediment in S4 and S15 are $21.2 \mu \mathrm{m}$ and $61.61 \mu \mathrm{m}$ respectively. For the relatively fine grain, there is an upward concave profile just above the upward convex profile (S4). For the relatively coarse grains, the curvature of the upward convex profile is smaller than for the fine grains. This is consistent with the conclusions in sheet flow condition by Absi (2006). This indicates that our model reflects the effect of sediment grain size.
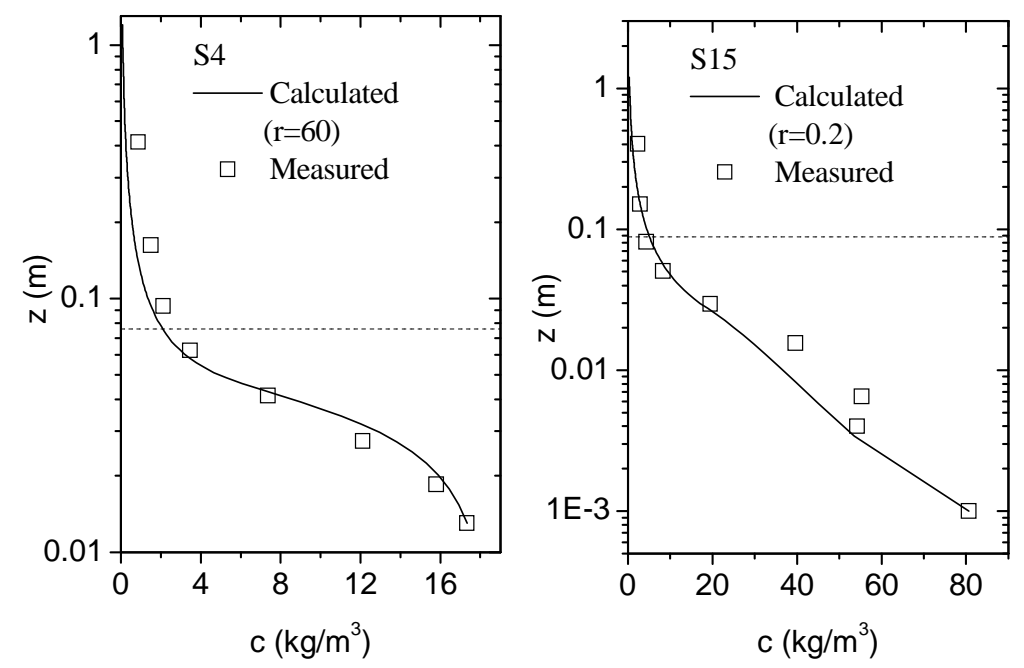

Figure 5. Comparison between the calculated results by the semi-theoretical model with measurements from experiments S4 and S15 of Lamb et al. (2004). 
The calculated results by the simplified model are also compared with measurements in Fig. 6, in which the good agreement is presented in the near-bed water. The shape of profiles predicted by the simplified model is similar with that by the semi-theoretical model. The concentrations above $0.2 \mathrm{~m}$ are still underestimated. This indicates that the combination of the finite-mixing-length theory and the damping function of diffusivity from traditional model is reasonable and effective.
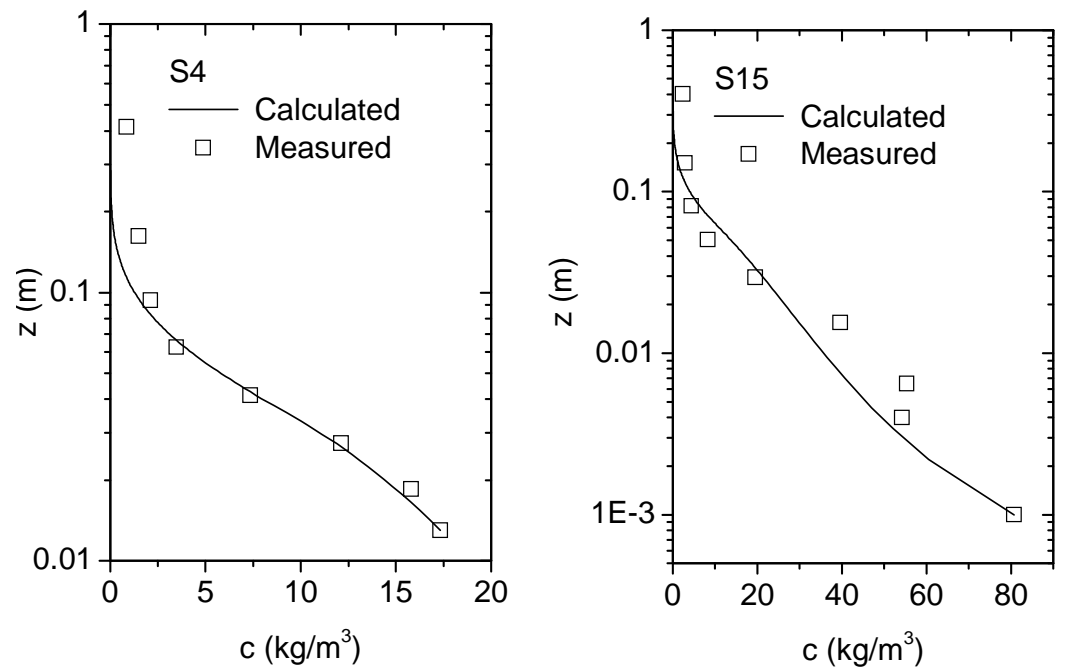

Figure 6. Comparison between the calculated results by the simplified model with measurements from experiments $\mathrm{S} 4$ and $\mathrm{S} 15$ of Lamb et al. (2004).

\section{CONCLUSIONS}

A semi-theoretical time-averaged suspended sediment concentration model for the HDS was developed based on the finite-mixing-length theory. We analyzed the data from U-tube experiments in Lamb et al. (2004) and Lamb and Parsons (2005) and found that the total turbulent intensity decreases exponentially with $z$ in HDS flow formed under waves as in the fresh open-channel flow but the friction velocity is weakened by the sediment-induced stratification. Because ratios of the distribution of turbulent energy with HDS can be taken as constant in three directions, the mixing velocity was derived using the vertical turbulent fluctuations. Another characteristic in the mixing process was that the mixing length was limited by the substantial suppression of vertical motion and the mixing length $l^{\prime}$ by Balmforth et al. (1998) was adopted.

Because the stability of the solution of the semi-theoretical model suffered from numerical oscillations, a simplified model was suggested. We combined the finite-mixing-length theory with the traditional model. The damping function of van Rijn's model (2007) was adopted to modify the apparent Fickian diffusivity derived from the finite-mixing-length theory. In this way we not only take advantage of the finite-mixing-length theory, but also simplify the calculation.

Finally, the calculated results by both the semi-theoretical model and the simplified model were compared with the measured concentration profile. Good agreement was found in the near-bed water. This indicated that the two models reflected the mechanisms of turbulent mixing for high-density suspension.

The turbulence-induced high-density suspension is a very complicated phenomenon. More credible data are needed to improve the models. In the future, we plan to formulate a time-averaged suspended sediment concentration model under waves for the whole water column based on the study of HDS in this paper.

\section{ACKNOWLEDGMENTS}

The research was supported by the Open Foundation of State Key Laboratory of Estuarine and Coastal Research, East China Normal University (Grant No. SKLEC200906).

\section{REFERENCES}

Absi, R. 2006. On the effect of sand grain size on turbulent mixing, Proceedings of $30^{\text {th }}$ International Conference on Coastal Engineering, ASCE, 3019-3029.

Balmforth, N.J., S.G.L. Smith and W.R. Young. 1998. Dynamics of interfaces and layers in a stratified turbulent fluid, Journal of Fluid Mechanics, 355, 329-358. 
Cheng, N.S. 1997. Effect on concentration on settling velocity of sediment particles, Journal of Hydraulic Engineering, 123, 728-731.

Han, H.S. 2005. Vertical profile of suspended fine sediment concentration under breaking waves, Master thesis, Tianjin University (in Chinese).

Hou, Z.Q. and H.Yang. 2004. Analysis of sudden siltation of outer channel of Huanhua Harbor, Journal of Waterway and Harbor, 25, 213-225 (in Chinese).

Lamb, M.P., E. D’Asaro and J.D. Parsons. 2004. Turbulent structure of high-density suspensions formed under waves, Journal of Geophysical Research, 109, C12026.

Lamb, M.P. and J.D. Parsons. 2005. High-density suspension formed under waves, Journal of Sedimentary Research, 75, 386-397.

Lewis, W.K., E.R Gilliland. and W.C. Bauer. 1949. Characteristics of fluidized particles, Indusrtial\& Engineering Chemistry, 41, 1104-1117.

Nezu, I. and H. Nakagawa. 1993. Turbulence in open channel flows, IAHR Monograph, Balkema, Rotterdam.

Nielsen, P. 1992. Coastal bottom boundary layers and sediment transport, World Scientific Publication, Singapore.

Nielsen, P. and I.A.L. Teakle. 2004. Turbulent diffusion of momentum and suspended particles: A finite-mixinglength theory, Physics of Fluids, 16, 2342-2348.

Swart, D.H. 1974. Offshore sediment transport and equilibrium beach profiles, Publication No. 131, Delft Hydraulic Laboratory.

Teakle, I.A.L. 2006. Coastal boundary layer and sediment transport modeling, Ph.D. Dissertation, Queensland University.

Traykovski, P., W.R. Geyer, J.D. Irish, and J.F. Lynch. 2000. The role of wave-induced density-driven fluid mud flows for cross-shelf transport on the Eel continental shelf, Continental Shelf Research, 20, 2113-2140.

van Rijn L.C. 2007. Unified view of sediment transport by currents and waves: suspended transport, Journal of Hydraulic Engineering, 133, 668-689.

Vinzon, S.B. and A.J. Mehta. 1998. Mechanism for formation of lutoclines by waves, Journal of waterway, port, coastal and ocean engineering, 124, 147-170.

Winterwerp, J.C. 2006. Stratification effects by fine suspended sediment at low, medium, and very high concentrations, Journal of Geophysical Research, 111, C05012.

You, Z.J., D.L. Wilkinson. and P. Nielsen. 1991. Velocity distribution of waves and currents in the combined flow, Coastal Engineering, 15, 525-543.

Zhao, Q. 2006. Fine sediment transport in storm event the Huanghua experience. Proceedings of $30^{\text {th }}$ International Conference on Coastal Engineering, ASCE, 2956-2968. 